\title{
Effect of Specimen Transport Network System in the Turnaround Time on Patients Tuberculosis Genexpert Test Results in the West Region of Cameroon
}

\section{Ngala Solange Mudih, Bih Adelaide, and Gamo Djouomo Francis}

\begin{abstract}
Introduction

Specimen transport network system is a major tool in getting quick turnaround time for patient results in general and for tuberculosis (TB) diagnosis in particular given that TB is an airborne disease and any mismanagement of its specimens, sputum in particular, can lead to generation of aerosols, hence spread and infection of the persons transporting the specimens and the health care personnel receiving the specimens and the delay in the diagnosis leading to more spread of the bacilli and up to the death of the patient.

It is therefore of great essence to establish a reliable specimen transport network system which is essential for effective TB patient care, allowing for rapid diagnosis, initiation of treatment and patient follow up in the West Region of Cameroon and Cameroon as a whole. This article is therefore aimed at establishing a better specimen transport network system to better the turn- around time for TB specimens in the West Region of Cameroon.

\section{Method}

A descriptive retrospective study by means of secondary data collection was carried from January 2016 to July 2017 on 1,130 specimens requested by clinicians before the GeneXpert instruments were introduced in the West Region of Cameroon and from October 2017 to July 2018 when two GeneXpert instruments were introduced in the West region. Request forms with date of test requested at the health facilities in the West region and results registers in which date results from the Reference Laboratory were communicated to health facility in the West Region, were used as the data sources to calculate the turn- around time (TAT). TAT was analyzed in hours and converted to days using excel.

Results

Results from this study shows an average decrease in turnaround time of patient's results from 26 days when the specimens were tested only at the Reference laboratory in Bamenda to 7 days when two GeneXpert instruments were introduced in the West region with a total of 2 platforms where tests could possibly be done.

\section{Conclusion}

Re designing a better specimen transport network system and increasing the number of Xpert platforms not only in the West region but in all other regions of Cameroon, will reduce TAT for TB results to only 01 to 02 days, thus increase quality of prevention and treatment programs, thereby reducing costs and live savings of the population.
\end{abstract}

Keywords: TB specimens, Transport network System. Turnaround time, Patients results

\section{LIST OF ABBREVIATIONS}

DBS: Dry Blood Spot

DTC: Diagnostic and Treatment Center 
GLI: Global Laboratory Initiative

HCW: Health Care Workers

HIV/AIDS: Human Immunodeficiency Virus/Acquired Immune Deficiency Syndrome

RR: Rifampicin Resistant

TAT: Turnaround time

TB: Tuberculosis

WHO: World Health Organization

\section{Introduction}

Tuberculosis (TB) is one of the top ten global causes of mortality and poses a particular acute threat to developing countries (WHO, 2016). Of the 1.8 million TB related deaths in 2015, more than $95 \%$ occurred in low and lower-middle income countries, due to weak case detection and treatment systems (WHO, 2016). Early and accurate detection of TB is important for the timely initiation of treatment and prevention of TB transmission (Kwak N, et al. 2013)

Specimen transport network system is a major tool in getting quick turnaround time for results (Charles Kiyaga et al. 2014), especially to specimens for the diagnosis of TB, reason being that TB is an airborne disease and any mismanagement of its specimens can lead to the generation of aerosols, hence infection of the persons transporting the specimens.

GeneXpert allows patient have full results-including TB and RR status in an average of one day (Charles Kiyaga et al, 2014). This allowed for improved patient contact and reduced risk of not returning to health facility to collect results or begin treatment. In low demand remote locations which allowed for same -day testing, (given the two-hour test cycle) patients could save an entire trip to the facility by waiting for results instead of returning another day (UNITAID, 2017).

Despite the guidelines proposed by WHO (WHO, 2015), GLI (GLI, 2017) and other organizations for good specimen transport network initiations in TB diagnostic sites, these guidelines are not implemented in most parts of Cameroon and especially in the West region. In Zimbabwe, USAID partnered with existing transport system (riders for health) to establish a reliable transport system for TB specimens (sputum) whereby a network of motorcycles was used to quickly deliver sputum specimens from clinics to be tested in laboratories. Due to this intervention, the time needed to provide an accurate TB diagnosis dropped from two to three weeks to only one to two days. (Charles Kiyaga et al. 2014)

In Cameroon, TB diagnosis using the GeneXpert instrument or culture is free due to the government subvention. But due to a poor specimen transport network system from healthcare centers and clinics to diagnostic centers in the West Region, most patients either got sicker or even died before getting their results, keeping the patient's caregivers and family frustrated and more at risk of developing the TB disease in the nearest future due to contact with the infected.

At the initiation of GeneXpert platform in Cameroon, all specimens eligible for GeneXpert testing for TB diagnosis from the West region from all 20 DTC (diagnostic and treatment centers) were sent to the Tuberculosis Reference Laboratory Bamenda- Cameroon. When TB specimens were received from patients at the DTC of the heath facilities in the West region, they were kept at the sites and one transporter would collect all the specimens from each of these 20 sites once a week or whenever he was available. The specimens were then piled in one flask and sent through a traveling agency to the TB reference lab in Bamenda for GeneXpert testing.

Specimens from the North West region, where the GeneXpert platform was, were being prioritized over those from other regions for testing, given that they came in direct contact with patients in the region unlike 
with just specimens from the West region that were sent through a travel agency. During days of heavy workload, specimens received from other regions were kept for several days in order to clear up all specimens from sites in the North West Region before processing those from West Region and or other regions.

In 2017, two GeneXpert platforms were introduced in the West region of Cameroon in two hospitals. The Government of the country provided reagents for free for the diagnosis of multidrug resistance TB for retreatment cases, prisoners, children and people living with HIV/AIDS.

Out of the 20 DTCs in the region, 16 send specimens to the Regional Hospital Bafoussam and 4, to the St. Vincent de Paul, Dschang and when there were difficult cases, the specimens were sent to the Tuberculosis Reference Laboratory Bamenda in Bamenda. This distribution is because of the proximity of these health facilities to where the GeneXpert platforms are located, with distance ranging from $30 \mathrm{~km}-130 \mathrm{~km}$ to testing sites. Even with these arrangements and increase in the Xpert platforms, patients still complained of not receiving their results within 1 to 2 days. This indicates that the specimen transport network system is not effective or reliable.

Before the introduction of the GeneXpert test instrument for TB diagnosis in the West region of Cameroon, turnaround time for test results was 26 days in which case some of the patients might have become critically ill or had died before getting their results.

It is of great essence to establish a reliable specimen transport network system which is essential for effective TB patient care, allowing for rapid diagnosis, initiation of treatment and patient follow up in the West region and the whole of Cameroon. This article is therefore aimed at establishing a better specimen transport network system to better the turn-around time for TB specimens in the West Region of Cameroon.

\section{Methodology}

A descriptive retrospective study by means of secondary data collection was carried out from January 2016 to July 2017 on 1,130 specimens before the GeneXpert instrument was introduced in the West region of Cameroon and from Oct 2017 to July 2018 on 2463 specimens when two GeneXpert instruments were introduced in the region. The effect of TAT from the time specimens were processed in Bamenda and in West was analyzed using excel and results reported in days.

TB registers and patients request forms and results registers were reviewed to obtain data before and after introduction of the GeneXpert instrument.

TAT was calculated in hours using excel and converted to days, from the time specimens were collected from the patient- indicated on the request forms in TB registers, to the time results were ready and available to be given back at the healthcare facility in the West region- seen in the results register.

\section{Results and Discussion}

From this review, with the introduction of two platforms and decentralization of the specimens to be tested, there was an average decrease in TAT from 26 days to Seven days. This TAT could further decrease to only one to two days with an effective specimen transport network system put in place. The following factors still persist and affect enormously the TAT of TB specimens in the region

The distance to health facilities where GeneXpert platforms are found is about 30-130km from other clinics and healthcare facilities, thereby requiring an effective and reliable specimen transport network system, given specimens take a long time to get to these Xpert sites. The distance of $130 \mathrm{~km}$ is that covered from specimens to leave some sites in the West region to the reference Laboratory in Bamenda. This long distance to get specimens to the lab for processing in another region gets the turnaround of 26 days and to seven days when specimens are being processed also in the West region using the two geneXpert platforms. This decrease to seven days is still a long time for patients to get their results and can be reduced to one to two days with an effective and reliable specimen transport network system to the diagnostic sites. 
Other factors affecting the TAT in the region include personnel workload. Laboratory personnel or health care worker assigned, amidst the workload at their facilities, need to organize themselves to transport these specimens for testing. This at times is not possible and specimens are piled till when staff are a little free to dedicate time for this activity.

To add, personnel need to finance transportation to and from the travel agencies for transportation to the testing sites. Since there are no transport vouchers for this, no reimbursement is done. Because of this, specimens are being piled in the healthcare facilities in order to get a reasonable number before sending to the testing sites, in order to reduce financial burden on the health care worker. To this effect, specimen quality is compromised during this time as most at times they are left at room temperature and the viability is lost. Testing sometimes were not done for most specimens after reaching the testing sites because either they are spilled or sputum have dried up completely due to improper packaging. When specimens do not meet the criteria for processing; they are rejected and the patients need to be called for recollection of specimen before sending for processing. Delays at all the levels greatly contribute to increased turnaround time and the patients are most affected in that no diagnosis is done and they cannot be further assessed by the clinician. Most patients have health worsen especially if the resistant mycobacterium tuberculosis bacteria were present and some even die before results were ever obtained. Also, personnel use their personal communication credits to follow up for the processing of specimens and reception of results. Results are therefore delayed sometimes to be known at the facility and so patients are not notified in time when results are ready, thus giving a false increase in TAT.

\section{Conclusion}

An Effective and reliable specimen transport network system greatly plays an important role in the health of patients, with TB for example which is airborne and has a high transmission rate, improving on the specimen transport network system will lead to a quick diagnosis, quick results and immediate placement on treatment, thus reducing transmission rate, improving patient health and cost savings.

\section{Recommendations}

Re designing the specimen transport network system in the West region to include new health facilities will go a long way to reduce the turnaround time for results.

\section{To the Government.}

- Additional Xpert platforms in the region will go a long way to reduce workload involved in processing of specimens for facilities and also further reduce the TAT as there will be decentralization of specimens.

- Installation of GxAlert software and training of health care workers for fast delivery of results will help reduced TAT.

- Synergy of programs (HIV/TB for example) to a common transport network system for specimen, given that for the HIV program, many bikers are recruited for transport of DBS from interior localities to urban health facilities.

\section{To the health facilities}

- Providing and training bikers for all DTCs for specimen transport to facilities having Xpert platform will help to create an effective and reliable transport network system

- Subsidize transport cost for all HCWs who go to send specimens through agencies. This can be done by providing transport vouchers and taxi logs which can be used as proves for reimbursement

- Providing communication credit to health care workers will help facilitate communication of results and follow up of sent specimens. 


\section{References}

[1] Charles Kiyaga et al (2014). Uganda's New National Laboratory Sputum Transport System: A Successful Model for Improving Access to Diagnostic Services for Early Infant HIV Diagnosis and Other Programs. PloS ONE. 2014; 9(1).

[2] (WHO, 2015), Guidance on regulation for the transportation of infectious substances 2015-1016. Geneva, World Health Organization.

[3] Kwak N, Choi SM, Lee J, Park YS, Lee C-H, Lee S-M, et al. (2013) Diagnostic Accuracy and Turnaround Time of the Xpert MTB/RIF Assay in Routine Clinical Practice . PloS ONE 8(10). E77456. doi:10.1371/ journal.pone.0077456

[4] Specimen transportation system: An innovative approach to intensifying TB diagnosis in Zimbabwe. http://www.usaid.gov : February 23,2016

[5] (GLI, 2017), TB specimen referral system and integrated network 2017. Global laboratory initiative, GLI.

[6] UNITAID (2017). UNITAID end-of-project evaluation: TB GeneXpert -Scaling up access to contemporary diagnostics for TB (2017)

[7] WHO, (2016) Global TB Report, 2016. 